\title{
Scoring and psychometric validation of the Perception of Anticoagulant Treatment Questionnaire (PACT-Q ${ }^{\odot}$ ) MH Prins ${ }^{1,2}$, I Guillemin*3, H Gilet ${ }^{3}$, S Gabriel ${ }^{4}$, B Essers 5 , G Raskob 6 and SR Kahn 7
}

\begin{abstract}
Address: ${ }^{1}$ The Department of Epidemiology, Care and Public Health Research Institutes, University of Maastricht, Maastricht, the Netherlands, ${ }^{2}$ Department of Clinical Epidemiology and Medical Technology Assessment, Academic Hospital, Maastricht, the Netherlands, ${ }^{3}$ Mapi Values, Lyon, France, ${ }^{4}$ Sanofi-Aventis, Paris, France, ${ }^{5}$ Department of Clinical Epidemiology and Medical Technology Assessment, University Hospital Maastricht, the Netherlands, ${ }^{6}$ Department of Medicine, University of Oklahoma Health Sciences Center, Oklahoma City, USA and ${ }^{7}$ Department of Medicine, McGill University Division of Internal Medicine, and Center for Clinical Epidemiology \& Community Studies Jewish General Hospital, Montreal, Canada
\end{abstract}

Email: MH Prins - Mh.Prins@EPID.unimaas.nl; I Guillemin* - iguillemin@mapi.fr; H Gilet - hgilet@mapi.fr; S Gabriel - Sylvie.Gabriel@sanofiaventis.com; B Essers - bes@ms-azm-1.azm.nl; G Raskob - Gary-Raskob@ouhsc.edu; SR Kahn - susan.kahn@mcgill.ca

* Corresponding author

Published: 7 April 2009

Health and Quality of Life Outcomes 2009, 7:30 doi:10.1 186/1477-7525-7-30
Received: 30 May 2008

Accepted: 7 April 2009

This article is available from: http://www.hqlo.com/content/7///30

(c) 2009 Prins et al; licensee BioMed Central Ltd.

This is an Open Access article distributed under the terms of the Creative Commons Attribution License (http://creativecommons.org/licenses/by/2.0), which permits unrestricted use, distribution, and reproduction in any medium, provided the original work is properly cited.

\begin{abstract}
Background: The 'Perception of Anti-Coagulant Treatment Questionnaire' (PACT-Q) was developed to assess patients' expectations of, and satisfaction with their anticoagulant treatment. This questionnaire needs to be finalised and psychometrically validated.

Methods: The PACT-Q was included in the United States, the Netherlands and France into three phase III multinational clinical trials conducted to evaluate efficacy and safety of a new long-acting anticoagulant drug (idraparinux) compared to vitamin $K$ antagonist (VKA). PACT-Q was administered to patients with deep venous thrombosis (DVT), atrial fibrillation (AF) or pulmonary embolism (PE) at Day I, to assess patients' expectations, and at 3 and 6 months to assess patients' satisfaction and treatment convenience and burden. The final structure of the PACT-Q (Principal Component Analysis - PCA - with Varimax Rotation) was first determined and its psychometric properties were then measured with validity of the structure (Multitrait analysis), internal consistency reliability (Cronbach's alpha coefficients) and known-group validity.

Results: PCA and multitrait analyses showed the multidimensionality of the "Treatment Expectations" dimension, comprising 7 items that had to be scored independently. The "Convenience" and "Burden of Disease and Treatment" dimensions of the hypothesised original structure of the questionnaire were combined, thus resulting in 13 items grouped into the single dimension "Convenience". The "Anticoagulant Treatment Satisfaction" dimension remained unchanged and included 7 items. All items of the "Convenience" and "Anticoagulant Treatment Satisfaction" dimensions displayed good convergent and discriminant validity. The internal consistency reliability was good, with a Cronbach's alpha of 0.84 for the "Convenience" dimension, and 0.76 for the "Anticoagulant Treatment Satisfaction" dimension. Known-group validity was good, especially with regard to occurrence of thromboembolic events within 3 months from randomisation.
\end{abstract}




\begin{abstract}
Conclusion: The PACT-Q is a valid and reliable instrument that allows the assessment of patients' expectations and satisfaction regarding anticoagulant treatment, as well as their opinion about treatment convenience of use. Its two-part structure - assessment of expectations at baseline in the first part, and of convenience, burden and treatment satisfaction in the second - was validated and displays good and stable psychometric properties. These results are not sufficient to recommend the use of satisfaction as primary endpoint in clinical trials; further validation work is needed to support the interpretation of PACT-Q dimension scores. However, this first validation makes the PACT-Q an appropriate measure for use in clinical and pharmacoepidemiological research, as well as in real-life studies.
\end{abstract}

Trial Registration: (ClinicalTrials.gov numbers, NCT00067093, NCT00062803 and NCT00070655).

\section{Background}

Oral vitamin $\mathrm{K}$ antagonists (VKA) are effective and commonly used anticoagulant agents for the secondary prevention of venous thromboembolic disease and the prevention of systemic embolism in patients with atrial fibrillation [1]. However, because of patients' monitoring requirements, their inherent limitations in daily life (e.g. diet and activities) and the considerable inter-individual variability in pharmacodynamic effect, the burden of VKA on patients' daily life is highly significant, especially when given as long-term therapy [2-4]. Together with possible side effects such as bleedings, anticoagulant treatment may negatively affect patients' health-related quality of life (HRQOL) and treatment satisfaction, which in turn is likely to result in the decrease of the treatment effectiveness and ultimately in its failure [5-9]. Patients' satisfaction with treatment mainly depends on their previous experiences of similar treatment to which they compare their new treatment, as well as on their expectations [10]. Thus, especially when clinical outcomes regarding treatment efficacy and tolerance are rare and undistinguishable between different treatments, information about patients' satisfaction with their treatment and HRQoL is highly valuable. Surprisingly, despite the large use of VKA, no specific questionnaire was available to assess patients' satisfaction with their treatment and to evaluate their unmet needs. Therefore, the Perception of Anti-Coagulant Treatment Questionnaire (PACT-Q) was developed [11] as a means to investigate the burden of disease in patients with deep venous thrombosis (DVT), pulmonary embolism (PE) or atrial fibrillation (AF), with specific focus on patients' satisfaction with anticoagulant treatment and treatment convenience. The questionnaire was administered in the United States, the Netherlands and France during international phase III clinical trials in patients with DVT, PE or AF. These studies aimed at evaluating the efficacy and safety of the new anticoagulant drug -idraparinux- injected subcutaneously once a week, compared to VKA. The trials were multicentre, international, randomised in two arms (VKA versus idraparinux), openlabel and assessor-blind.
In order to be fully acceptable as a meaningful endpoint in clinical trials, the structure of a questionnaire has to be validated, its scoring rules established and its psychometric properties demonstrated. In this paper, we present the finalisation, i.e. scoring and validation of the original structure of the questionnaire. The psychometric validation of the resulting dimensions is also presented.

\section{Methods}

\section{The original PACT-Q}

The original PACT-Q consists of two parts and contains 27 items [11]: the PACT-Q1, composed of a single dimension (7 items), covering the expectations of patients regarding their anticoagulant treatment, is to be administered before treatment initiation; the PACT-Q2, composed of 3 dimensions covering the convenience of use of the treatment (11 items), burden of disease and treatment (2 items) and satisfaction with the anticoagulant treatment ( 7 items) as perceived by the patient, is to be administered to patients once the treatment is ongoing [11]. All the items of PACT-Q1 and PACT-Q2 parts are to be answered on a 5-point Likert scale. The questionnaire was simultaneously developed in French, American English and Dutch following a rigorous development process, in which the data collected from the patients were given particular importance [11]. It was further translated and adapted into 11 different country-specific versions following recommended linguistic validation procedures that included forward and backward translations by native speakers [12].

\section{PACT-Q administration}

The PACT-Q was administered during phase III clinical trials (DVT Clinical Study Protocol 64717/EFC3491; PE Clinical Study Protocol 64714/EFC 3484; AF Clinical Study Protocol 64720) as secondary endpoint in multicentre studies conducted in Europe (Austria, Belgium, Czech Republic, Denmark, France, Germany, Italy, the Netherlands and Poland), North America (Canada and the United States) and Oceania (Australia and New Zealand), with patients with DVT, AF or PE. All three trials 
were randomised, open-label and assessor-blind, and were conducted in accordance with the principles stated in the Declaration of Helsinki. Patients who provided an oral consent prior to their inclusion participated in the study. At Day 1, patients were asked to complete the PACT-Q1 part of the questionnaire; at 3 month (M3) and 6 month (M6), patients were asked to complete the PACTQ2 part.

\section{Definition of the study populations}

Patients included in the analyses had at least one PACTQ1 and one PACT-Q2 completed and assessable (i.e. 50\% of items completed) at Day 1 and M3, respectively, and did not violate the protocol for PACT-Q administration along the whole study (Figure 1). As the PACT-Q was originally developed in French, American English and Dutch, only patients from France, the United States and the Netherlands were included in the study populations. In order to control learning bias and for the purpose of the validation [13], two populations were pre-specified: the "scoring and initial validation" population subset was constituted of 452 patients with either DVT, AF or PE and from either the United States, the Netherlands or France; the "robustness" population subset was constituted of 200 AF patients from either the United States or the Netherlands. The "pooled" population $(n=652)$, corresponding to the "scoring and initial validation" population subset combined with the "robustness" population sub-

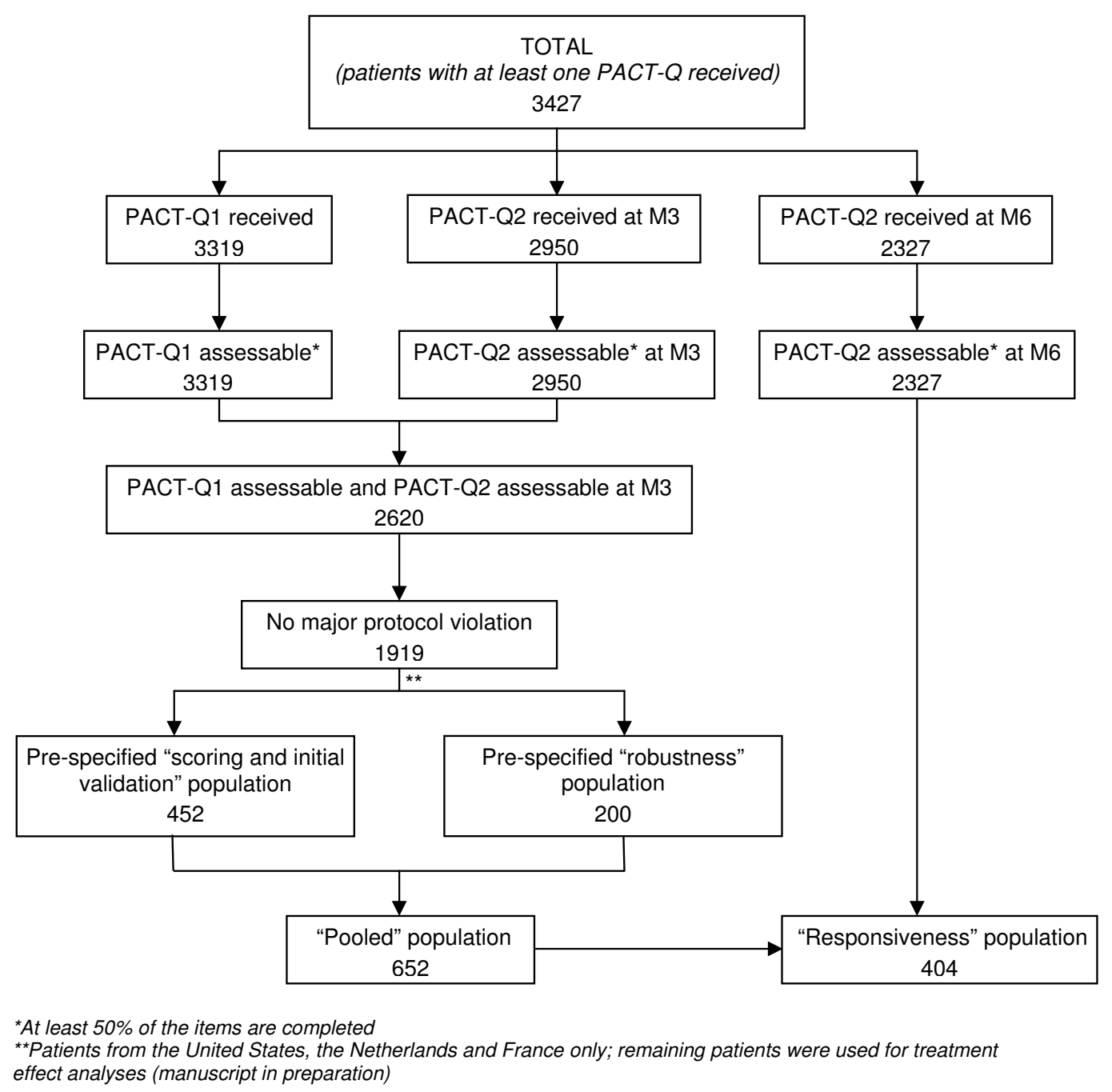

Figure I

Flowchart of the population included and participating in the study. 
set, was used to determine the PACT-Q psychometric properties. Patients who answered at least $50 \%$ of the PACT-Q2 items at M6 were included in the responsiveness analysis of PACT-Q2 ("responsiveness" population subset).

\section{Statistical analyses}

One should note that although the clinical trial was openlabel, statistical analyses were blind to treatment attribution, i.e. no distinction was made between the treatment arms that patients were randomly allocated to.

Finalisation: scoring and initial validation of PACT-Q original structure Principal Component Analysis (PCA) with Varimax Rotation is often used to assess the hypothetical structure of an instrument, by observing how the different items are spontaneously grouped into factors [14]. In our case, PCA was performed to check whether the factorial structure of PACT-Q reproduces the number and the type of dimensions that were hypothesised during the development step. Multitrait analysis (MA) defining the item convergent validity criterion (correlation between each item and its own dimension is considered satisfactory if it achieves $\geq 0.40$ ) and the item discriminant validity criterion (each item should have a higher correlation with its own dimension than with the other dimensions) was performed to ascertain the consistency of the dimensions [15].

The internal consistency reliability of the dimensions [16], i.e. the extent to which individual items are consistent with each other and reflect a single underlying construct, was assessed by the determination of Cronbach's alpha coefficient [17]. A value of 0.70 or above is recommended for group comparisons [18]. A Multiple Factorial Analysis (MFA) was carried out to analyse the relationship between PACT-Q1 and PACT-Q2 [19]. MFA assesses several sets of variables defined on the same set of individuals, and allows computation of a summary statistic referred to as the RV coefficient (vector correlation coefficient) [20]. RV value ranges from 0 to 1 ; the closer to 1 the $\mathrm{RV}$, the more similar the configuration of the two sets of variables.

\section{Validation and assessment of the psychometric properties of the $P A C T-Q$ dimensions}

Validity is the degree to which the instrument measures what it is supposed to measure [21-23]. Several types of validity were assessed. The validation of the conceptual model was assessed by performing MA with description of item convergent and discriminant validity [15]. Scalescale correlation was evaluated in order to test the construct validity of the questionnaire. Floor and ceiling effects were measured to check that there was no issue related to a high percentage of patients having the lowest or the highest possible score on any one scale. Clinical validity evaluates the extent to which the questionnaire is able to detect variability amongst patients with different clinical severity levels. Known-group validity can be evaluated when differences in scores are expected between groups of patients that differ on relevant variables. Clinical and known-group properties were assessed amongst groups of patients defined according to age, gender, international normalized ratio (INR - patients at risk of embolism, INR $<2$; patients with less risk of embolism, INR > $3)$, prior medication, thromboembolic events within the 3 months following randomisation, and time they spent in the 2-3 INR range between randomisation and M3.

Internal consistency reliability of the dimensions of the PACT-Q was again evaluated, by determination of the Cronbach's alpha coefficient [17]. Responsiveness refers to the ability of a questionnaire to detect important changes over a period of time [24]; it was assessed for the "Convenience" and "Anticoagulant Treatment Satisfaction" dimensions of the PACT-Q2 over M3 and M6, by determining the Effect-Size (ES) and Standardised Response Mean (SRM) [25-27]. A Wilcoxon signed rank test was performed in order to compare the change to 0 . A Kruskal-Wallis test was performed to test the hypothesis that the change in scores was significantly different across the different groups of patients. The PACT-Q responsiveness was tested for groups of patients defined according to the primary efficacy outcome (i.e. thromboembolic event within 3 months from randomisation consisting in stroke or a non-central nervous system systemic embolism for AF patients, and a PE or DVT event for PE and DVT patients respectively) and the time spent in the 2-3 INR range between randomisation and M6 visits. The threshold for statistical significance was set at 0.05 .

MFA was performed using SPAD Software. All the other data processing and analyses were performed with SAS Software for Windows (Statistical Analysis System, version 9).

\section{Results}

\section{Population characteristics}

Socio-demographic and clinical characteristics of the "pooled" population set that was used in the analyses are summarised in Table 1. The "pooled" population consisted of 652 patients, amongst whom 234 were from the United States, 309 from the Netherlands and 109 from France. Of these 652 patients, 426 patients had AF, 87 had DVT and 139 had PE. The mean age was 65 years, patients with AF being the oldest population (mean $=68$ yearsold). Overall, males were more represented than females, with the greatest difference observed within the AF population (70\% men). 
Table I: Socio-demographic and clinical characteristics of the "pooled" population at day I (n = 652)

\begin{tabular}{|c|c|c|c|c|c|c|c|c|c|c|c|}
\hline \multirow[t]{2}{*}{ Medical condition } & \multirow[t]{2}{*}{ Number of patients } & \multicolumn{3}{|c|}{ Country $^{a}(n)$} & \multirow{2}{*}{$\begin{array}{c}\text { Age } \\
\text { (years } \pm \text { SD) }\end{array}$} & \multicolumn{2}{|c|}{ Gender (\%) } & \multicolumn{3}{|c|}{ INR $^{\mathrm{b}, \mathrm{c}}(\mathrm{n})$} & \multirow{2}{*}{$\begin{array}{l}\text { Patients with prior } \\
\text { medication }(n)\end{array}$} \\
\hline & & US & NL & $\mathbf{F R}$ & & Male & Female & $<2$ & {$[2 ; 3]$} & $>3$ & \\
\hline Atrial fibrillation & 426 & 152 & 251 & 23 & $68 \pm 9.5$ & 70 & 30 & 152 & 207 & 64 & 72 \\
\hline $\begin{array}{l}\text { Deep venous } \\
\text { thrombosis }\end{array}$ & 87 & 58 & 0 & 29 & $54.6 \pm 16.8$ & 48 & 52 & 77 & 0 & 0 & 0 \\
\hline $\begin{array}{l}\text { Pulmonary } \\
\text { embolism }\end{array}$ & 139 & 24 & 58 & 57 & $59.9 \pm 16.4$ & 52 & 48 & 11 & 1 & 0 & 0 \\
\hline
\end{tabular}

a US, The United States; NL, The Netherlands; FR, France

b INR, International Normalized Ratio; MD

cMD, missing data $=39$

d Patients who had already received antithrombotic agents prior to participation in the study

\section{Challenging the original PACT-Q structure}

The analyses were performed with the "scoring and initial validation" population subset $(\mathrm{n}=452)$, including patients with AF $(n=226)$, DVT $(n=87)$ and PE $(n=139)$ from France $(n=109)$, the Netherlands $(n=209)$ and the United States $(\mathrm{n}=134)$.

Finalisation of the PACT-Q: initial validation of the original structure and scoring

The PCA with Varimax Rotation and MA were conducted on the PACT-Q1 completed at Day 1. The PCA performed on the 7 items of PACT-Q1 resulted in the definition of 2 factors with eigenvalues greater than 1, accounting for only 23\% (Factor 1, containing items A3, A5 and A7) and $18 \%$ (Factor 2, containing items A1, A2, A4 and A6) of the total variance. Correlations between the 7 items were very low, with Pearson coefficients ranging from -0.068 to 0.268 , indicating that the items were non-redundant. Cronbach's alpha was low (0.43). Item-dimension correlation coefficients ranged from 0.12 to 0.29 , reflecting low item convergent validity criteria. Taken together, these data indicated that the "Treatment Expectations" dimension was not unidimensional. Each of the items of this dimension will therefore be analysed separately.

Following PCA analysis with Varimax Rotation conducted on the PACT-Q2 part completed at M3, four factors were retained based on an eigenvalue greater than one, representing $53 \%$ of the total variance. Factor 1 contained all items of the original "Convenience" dimension (i.e. B1 to B9), except B10 and B11; factor 2 contained items D4 to D7 of the original "Anticoagulant Treatment Satisfaction" dimension; factor 3 the items B10, B11 of the "Convenience" dimension and the items C1 and C2 of the "Burden of Disease and Treatment" dimension; factor 4 the items D1, D2 and D3 of the original "Anticoagulant Treatment Satisfaction" dimension. MA results showed good item convergent validity criteria for all the items within their own respective dimension, except items B10 and B11 ("Convenience" dimension) and items D2 and D3 ("Anti- coagulant Treatment Satisfaction" dimension). All items reached the discriminant validity criterion, i.e. all items shared a higher correlation with their own dimension than with the other dimensions of the questionnaire. Cronbach's alpha was satisfactory for the "Burden of Disease and Treatment" dimension (0.66), and good for the "Anticoagulant Treatment Satisfaction" and the "Convenience" dimensions ( 0.79 and 0.82 , respectively). No ceiling effects were reported, whereas a floor effect was observed for the "Burden of Disease and Treatment" (57\%) and "Convenience" (26\%) dimensions.

To improve the measurement model of the PACT-Q2, several alternative models were further tested for their structure, amongst which one model was retained as it displayed the most satisfactory and stable properties. The retained model included 2 dimensions ("Convenience", containing all B and C items, and "Anticoagulant Treatment Satisfaction" dimension, containing all D items). All the items within each of the PACT-Q2 dimensions met the convergent validity criterion (ranging from 0.42 to 0.66 ), except items B10 and B11, and items D2 and D3. All items met the item discriminant validity criterion. A floor effect was still observed for the "Convenience" dimension; no ceiling effect was noted for the 2 dimensions. The respective Cronbach's alpha values were 0.83 and 0.79 for the "Convenience" and "Anticoagulant Treatment Satisfaction" dimensions. The correlation coefficient between the 2 dimensions was low (-0.25), confirming that they clearly covered two separate concepts.

Thus, the final PACT-Q2 was constituted of 2 dimensions: the "Convenience" dimension, comprising items B1 to B11 (from the original "Convenience" dimension) combined with items C1 and C2 (from the original "Burden of Disease and Treatment" dimension), and the "Anticoagulant Treatment Satisfaction" dimension containing items D1 to D7 (Figure 2). The structure, dimensions and detailed contents of the items of the final version of PACT$\mathrm{Q}$ are reported in Table 3 . 


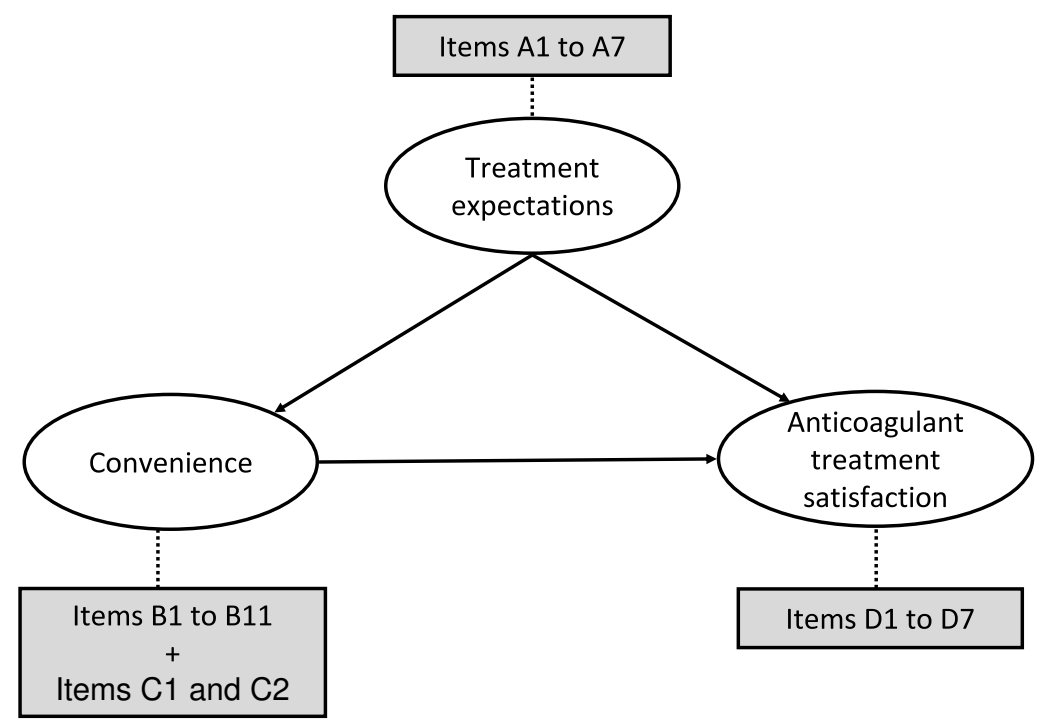

\section{Figure 2}

Conceptual framework of the final PACT-Q.

The final PACT-Q1 remained similar to the original version, i.e. containing 7 items, each individually scored from 1 to 5; for items A1, A2, A4 and A5, the higher the score, the higher the patients' expectations of their treatment; for items A3, A5 and A7, the lower the score, the higher the patients' expectations of their treatment (Table 2). Within the PACT-Q2, items B and C are reversed, summed and rescaled on a 0-100 scale to obtain the "Convenience" dimension score (Table 2). The higher the score, the higher the convenience and the lower the burden as perceived by the patient for their treatment. Items $\mathrm{D}$ are summed and rescaled on a $0-100$ scale to determine the "Anticoagulant Treatment Satisfaction" dimension score (Table 2); the higher the score, the higher the patient satisfaction with their anticoagulant treatment.

Studies of the link between PACT-QI and PACT-Q2

Pearson correlation coefficients between PACT-Q1 and PACT-Q2 (final structure) were below 0.20 for the majority of the items, indicating a weak relationship between the "Treatment Expectations" items and those assessing the "Convenience" and "Anticoagulant Treatment Satisfaction". RV coefficients between PACT-Q1 at Day 1 and PACT-Q2 at M3 were also low, ranging from 0.032 to 0.040, showing weak links between the dimensions of the two PACT-Q parts.

Validation and psychometric properties of the final PACT$Q$ dimensions

MA was first conducted with the "scoring and initial validation" population subset $(n=452)$. In order to use a sub- set of patients that had not been used for the scoring, the robustness of the PACT-Q structure was then validated with the "robustness" population subset $(n=200)$. A further analysis with the "pooled" population set $(n=652)$ allowed the validation to be consolidated.

Regardless of the country, the quality of completion of PACT-Q1 at Day 1 was good in the "pooled" population, with $1.1 \%$ missing data. For PACT-Q2, completion was still good though slightly lower, with $2.4 \%$ missing data at $\mathrm{M} 3$, and $1.5 \%$ missing data at M6.

Percentages of responses allocated to each of the response choices at Day 1 for the "Expectations" items are represented on Figure 3. The majority of patients answered "A lot" or "Extremely" for items A1, A2, A4 and A6. The majority of patients answered "Not at all" or "A little" for items A3, A5 and A7. Scores of the "Convenience" and "Anticoagulant Treatment Satisfaction" at M3 and M6 are summarised in Table 4. "Convenience" scores decreased from 91.3 at M3 to 90.6 at M6; "Anticoagulant Treatment Satisfaction" scores slightly increased from 68.9 to 70.6 , respectively.

Item convergent validity, floor and ceiling effects and internal consistency reliability of the PACT-Q2 dimensions obtained for these two subsets of population and the "pooled" population are summarised in Table 4. 
Table 2: Dimensions, detailed concepts and scoring method of the final PACT-Q

\begin{tabular}{|c|c|c|c|c|}
\hline PACT-Q & Dimensions & Item number - Detailed concept & Scoring & Score range \\
\hline \multirow[t]{7}{*}{ PACT-QI } & Treatment Expectations & AI - Confidence in prevention of blood clots & One score & I to 5 \\
\hline & & A2 - Expectations of symptom relief & One score & I to 5 \\
\hline & & $A 3$ - Expectations of side effects & One score & I to 5 \\
\hline & & A4 - Importance of ease of use & One score & I to 5 \\
\hline & & A5 - Worries about making mistakes & One score & I to 5 \\
\hline & & A6 - Importance of independency & One score & I to 5 \\
\hline & & A7 - Worries about cost & One score & I to 5 \\
\hline \multirow[t]{20}{*}{ PACT-Q2 } & Convenience* & $\mathrm{BI}$ - Difficulties in taking the treatment & One score & 0 to 100 \\
\hline & & B2 - Bother in taking the treatment & & \\
\hline & & B3 - Difficulties regarding dose adjustments required & & \\
\hline & & B4 - Treatment and other medications & & \\
\hline & & B5 - Treatment and regimen implications & & \\
\hline & & B6 - Treatment and being away from home & & \\
\hline & & B7 - Difficulties regarding daily life & & \\
\hline & & B8 - Bother in follow-up required & & \\
\hline & & B9 - Difficulties regarding regular intake & & \\
\hline & & BIO - Feeling regarding loss of independency & & \\
\hline & & BII - Worries about having to stop the treatment & & \\
\hline & & $\mathrm{Cl}$ - Impact of side effects on usual activities & & \\
\hline & & $\mathrm{C} 2$ - Discomfort due to symptoms & & \\
\hline & Anticoagulant Treatment Satisfaction* & DI - Feeling of reassurance & One score & 0 to 100 \\
\hline & & D2 - Symptom decrease & & \\
\hline & & D3 - Experience with side effects & & \\
\hline & & D4 - Satisfaction regarding independency & & \\
\hline & & D5 - Satisfaction with patient management & & \\
\hline & & D6 - Satisfaction with treatment form & & \\
\hline & & D7 - Overall satisfaction & & \\
\hline
\end{tabular}

\footnotetext{
* For convenience score, all items are reversed (reversed item score $=6-$ initial item score), then summed and rescaled on a $0-100$ scale; for satisfaction score, all items are summed and rescaled on a $0-100$ scale; for both scores, the higher the score, the higher the convenience/ satisfaction
}

Item convergent and discriminant validity criteria

Overall (i.e. in the "pooled" population), all the items within the "Convenience" dimension, except items B10 and B11, met the item convergent criteria and ranged from 0.33 to 0.64 . All the items within the "Anticoagulant Treatment Satisfaction" dimension met the convergent criterion, except items D2 and D3, and ranged from 0.33 to 0.63 . All the items of the 2 dimensions reached the item discriminant validity criterion.

Scale-scale correlation

The correlation coefficient between the "Convenience" and "Anticoagulant Treatment Satisfaction" dimensions of the PACT-Q2 was moderate (0.29), reflecting a fair relationship and no redundancy between the 2 dimensions.

\section{Floor and ceiling effects}

No floor effect was observed for either dimension of the PACT-Q2. No ceiling effect was noted for the "Anticoagulant Treatment Satisfaction" dimension, contrary to the "Convenience" dimension (percentage of patients at the highest possible score $=22 \%$ ).

Internal consistency reliability

Good internal consistency reliability was displayed within each of the PACT-Q2 dimensions, with Cronbach's alpha

Table 3: Description of PACT-Q2 dimension scores at M3 and M6

\begin{tabular}{|c|c|c|c|c|c|}
\hline & PACT-Q2 dimensions & $\mathbf{N}$ & Mean (STD) & Median (QI - Q3) & Min - Max \\
\hline \multirow[t]{2}{*}{ M3 } & Convenience & 651 & $91.3(10.4)$ & $94.2(88.5-98.1)$ & $32.7-100.0$ \\
\hline & Anticoagulant Treatment Satisfaction & 641 & $68.9(17.0)$ & $67.9(57.1-79.2)$ & $0.0-100.0$ \\
\hline \multirow[t]{2}{*}{ M6 } & Convenience & 404 & $90.6(10.4)$ & $94.0(86.5-98.1)$ & $23.1-100.0$ \\
\hline & Anticoagulant Treatment Satisfaction & 403 & $70.6(17.4)$ & $71.4(60.7-82.1)$ & $0.0-100.0$ \\
\hline
\end{tabular}


Table 4: Psychometric validation of the PACT-Q2 dimensions for the 3 population subsets

\begin{tabular}{|c|c|c|c|c|}
\hline Population subsets & Cronbach's alpha & Floor effectc (\%) & Ceiling effect ${ }^{d}(\%)$ & $\begin{array}{c}\text { Convergent and discriminant } \\
\text { validity criterion } \\
\text { (range of item-scale correlations) }\end{array}$ \\
\hline \multicolumn{5}{|c|}{ "scoring and initial validation" ( $n=452)$} \\
\hline Convenience & 0.83 & 0 & 21.2 & $0.31-0.63$ \\
\hline $\begin{array}{l}\text { Anticoagulant Treatment } \\
\text { Satisfaction }\end{array}$ & 0.79 & 0.6 & 4.0 & $0.36-0.66$ \\
\hline \multicolumn{5}{|l|}{ "robustness" (n = 200) } \\
\hline Convenience & 0.87 & 0 & 24.1 & $0.40-0.67$ \\
\hline $\begin{array}{l}\text { Anticoagulant Treatment } \\
\text { Satisfaction }\end{array}$ & 0.70 & 0 & 1.9 & $0.26-0.59$ \\
\hline \multicolumn{5}{|l|}{ "pooled" (n = 652) } \\
\hline Convenience & 0.84 & 0 & 22.1 & $0.33-0.64^{a}$ \\
\hline $\begin{array}{l}\text { Anticoagulant Treatment } \\
\text { Satisfaction }\end{array}$ & 0.76 & 0.4 & 3.3 & $0.33-0.63^{b}$ \\
\hline
\end{tabular}

a except items $\mathrm{BIO}$ and $\mathrm{BI}$, all items meet the convergent validity criterion

b except items D2 and D3, all items meet the convergent validity criterion

c percentage of patients with the lowest possible score (i.e. 0), that is patients who answered the least favourable response choice to all items of the dimension.

dpercentage of patients with the highest possible score (i.e. 100), that is patients who answered the most favourable response choice to all items of the dimension.

values of 0.84 for "Convenience", and 0.76 for "Anticoagulant Treatment Satisfaction".

\section{Known-group validity}

Scores of items A2, A4, A5 and A7 within the "Treatment Expectations" dimension at Day 1 (PACT-Q1), and the "Convenience" and "Anticoagulant Treatment Satisfaction" dimensions at M3 showed significant differences when compared according to the age of the patients: the highest scores of "Treatment Expectations" items were observed for patients under 60 years of age (data not shown). Patients aged between 60 and 65 displayed higher "Anticoagulant Treatment Satisfaction" scores than younger and older patients, this difference being significant $(\mathrm{p}=0.028)$. For the "Convenience" dimension, the highest score was observed for patients aged between 65 and 75 years $(\mathrm{p}<0.0001)$. Only the A2, A3 and A7 item scores of "Treatment Expectations" were significantly different between females and males, while other scores were similar over the 2 groups. When compared according to experience of prior medication, scores of the "Convenience" dimension were significantly higher for patients who did not have prior medication than patients who did (score of 92 versus 89, respectively; $\mathrm{p}=0.01$ ). A higher score was reported at item A2 ("Treatment Expectations" - symptom relief) for patients with no prior medication experience than for patients with experience (3.3 versus $2.8, \mathrm{p}=0.0044)$. In contrast, a significantly higher score for item A3 ("Treatment Expectations" - side effects) was observed for patients with prior experience of medication than patients with no prior medication ( 2.9 versus 2.4 , $\mathrm{p}$
$=0.0003)$. Scores in the "Anticoagulant Treatment Satisfaction" dimension were higher for patients who reported no events within 3 months of randomisation than for those who reported having had an event (69 versus 41, respectively; $\mathrm{p}=0.005$ ).

When compared between groups of patients defined according to their baseline INR level at Day 1 (i.e. $<2$, $[2 ; 3],>3$ ), scores of most items of PACT-Q1 (item A2, "symptom relief"; item A3, "side effects"; A4, "ease of use"; A5, "making mistakes"; and A7, "cost of the treatment") were significantly higher for patients with INR $<2$ than for patients with INR $=2$. Regarding PACT-Q2 at M3, the "Anticoagulant Treatment Satisfaction" score was the lowest for patients with an INR > 3, and significantly increased as INR decreased (scores ranging from 61 to 69). These results at M3 concerned only patients treated with VKA as the INR was not available after Day 1 for patients treated with the new anticoagulant treatment. None of the other differences observed between scores of the PACTQ2 dimensions or PACT-Q1 items were significant.

Similarly, score differences reported between groups of patients defined according to the time they spent in the 23 INR range (4 groups defined: $<50 \%$, between $50 \%$ and $60 \%$, between $60 \%$ and $70 \%$, and $\geq 70 \%$ ) were not significant. In other terms, the time spent in the 2-3 INR range between randomisation and M3 does not have a statistically significant impact on scores. Again, these results concerned only patients treated with VKA, as those treated 


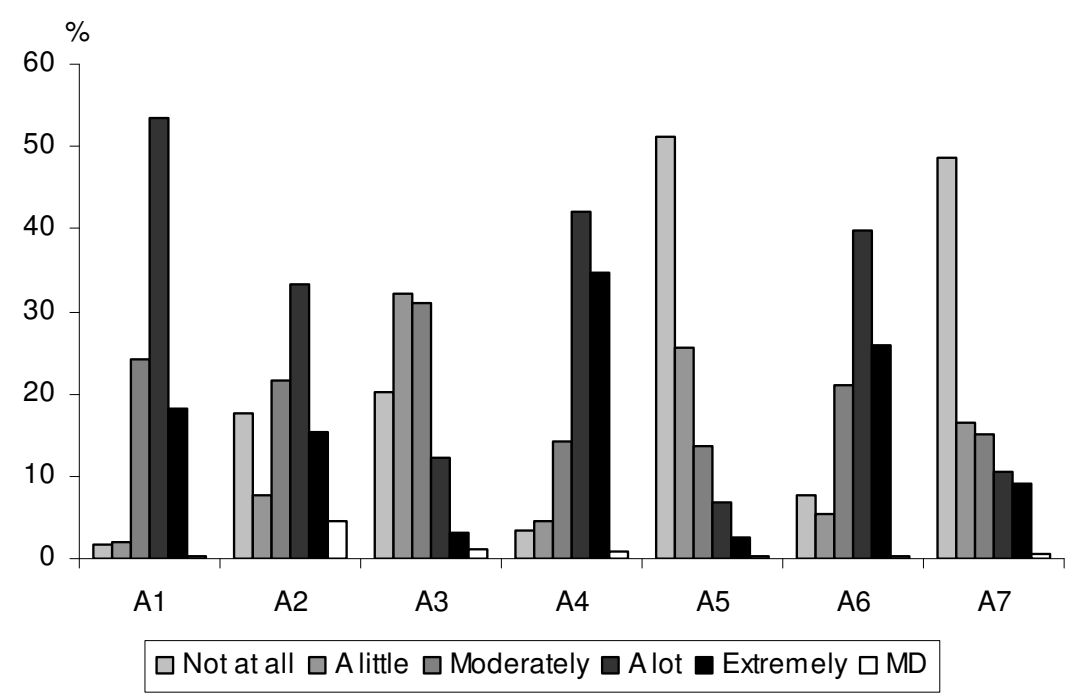

Content of PACT-Q1 items :

A1 - Confidence in prevention of blood clots

A2 - Expectations of symptom relief

A3 - Expectations of side effects

A4 - Importance of ease of use

A5 - Worries about making mistakes

A6 - Importance of independency

A7 - Worries about cost

MD, missing data

Figure 3

Description of the percentage of patients per response choice to the "Expectations" items at Day I ( $\mathrm{N}=652)$.

with idraparinux did not have INR monitoring during the study.

\section{Change of PACT-Q2 scores over 3 months}

Overall, no significantly different changes in the "Convenience" and "Anticoagulant Treatment Satisfaction" scores were observed from M3 to M6, whether compared between groups of patients defined according to their report of a thromboembolic event (or not) within 3 months of randomisation, the time they spent within the 2-3 INR range between randomisation and $M 3$, and M3 and M6, or the impact of the INR control status deterioration or improvement. Most of the changes in scores observed within each group were not significantly different to 0 ( $\mathrm{p}$-signed rank test $>0.05$ ).

\section{Discussion}

The PACT-Q was included as a secondary endpoint in phase III clinical trials in order to assess the expectations and satisfaction of patients suffering from DVT, PE or AF and treated with a new long-acting anti-thrombotic drug injected once a week subcutaneously, in comparison with an oral VKA treatment. The finalisation and psychometric validation steps of the questionnaire were performed using the patients of the clinical trials.

\section{Validity of the original PACT-Q structure}

Regarding the PACT-Q2 part, the exploratory PCA performed on the original PACT-Q revealed a structure very close to the proposed original structure [11], with 4 factors covering treatment convenience (items B1 to B9), treatment satisfaction (items D4 to D7), burden of disease and treatment and convenience of use (items C1 and C2 and items B10 and B11, respectively), and patients' anticoagulant treatment satisfaction (items D1 to D3). The overall good homogeneity of the PACT-Q2 dimensions, as reflected by the Cronbach's alpha values of 0.79 ("Anticoagulant Treatment Satisfaction" dimension) and 0.82 ("Convenience" dimension), confirmed the well-founded hypothesis of the original structure of the questionnaire. The lower value (0.66) reported for the "Burden of Disease and Treatment" dimension is perfectly acceptable as it contains only 2 items. As for the PACT-Q1 part, the weak correlations between each of the items and their low item convergent validity criterion, together with the low internal consistency reliability of the "Treatment Expecta- 
tions" dimension, strongly suggests that the items of PACT-Q1 cannot be thought of as one single concept.

\section{Scoring and psychometric validation of the PACT-Q}

The last series of PCA and MA led to the conclusion that the 7 items of the "Treatment Expectations" of PACT-Q1 were to be scored individually. This is not surprising as expectations cover heterogeneous concepts, each of them being measured by a single item. After the last finalisation step, the PACT-Q2 was reduced from 3 to 2 dimensions covering treatment "Convenience" (13 items) and "Anticoagulant Treatment Satisfaction" ( 7 items). The number of items in the 2 dimensions is quite high, and one could wish to reduce the PACT-Q to a short form. However, no items were removed as they were all relevant for the purpose of the questionnaire and the concepts were shown to be pertinent and well-accepted by patients. The use of PACT-Q in further clinical studies would be needed to definitively confirm their relevance. The reliability of these findings is increased by the use of separate subsets of population for the scoring and initial validation and for the psychometric validation.

The good quality of completion and returns of both PACT-Q1 and PACT-Q2 in all of the countries reflected the good acceptability of the questionnaire. The PACT-Q2 structure was further confirmed by a good item convergent and discriminant validity criterion and excellent internal consistency reliability of both dimensions. No floor or ceiling effects were observed for the "Anticoagulant Treatment Satisfaction" dimension; in contrast, a ceiling effect (22\%) was reported for the "Convenience" dimension. While a ceiling effect is not recommended when measuring quality of life aspects [21], it is easily conceivable and acceptable that a majority of patients report no inconvenience of use in their treatment, particularly in clinical trials.

Known-group validity showed that PACT-Q dimensions were able to discriminate between groups of patients presenting different disease experiences and characteristics. In particular, patients who had had no previous experience of anticoagulant treatment were significantly more demanding in terms of symptom relief, while patients who had already been treated with anticoagulants expected more side effects than those who had not, and could therefore possibly be more realistic. These findings agree with Oliver's work, who proposed that patients' expectations are considerably influenced by previous experiences and knowledge that they have acquired of their disease and treatment [10]. As expected, patients' satisfaction was greater if they did not have a thrombolic event within the period of time that they were under treatment. In addition, one should point out that although a few patients only $(n=4)$ experienced an event within these 3 months, this had a noticeable impact on their satisfaction, thus indicating that PACT-Q is sensitive to events that are meaningful to patients. Lastly, patients at risk of embolism (INR $<2$ ) at Day 1 of the study expected much more from their anticoagulant treatment in terms of cost, ease of use, side effects, worries about making mistakes and symptom relief, when compared to patients with lower risk of embolism (INR > 3). After 3 months of anticoagulant treatment, patients with an INR $>3$ (i.e. with less risk of embolism) were the most satisfied with their treatment compared to patients with a higher INR. Interestingly, no correlation could be drawn between patients' expectations from the anticoagulant treatment at baseline and their satisfaction after 3 months of treatment. This can be explained by the theoretical model of Oliver et al., in which the authors propose that patients derive their satisfaction from the comparison between their expectations before starting the treatment and the performance they eventually perceive from it after being treated [10]. Therefore, in order to study the link between expectations and satisfaction, it would be necessary to assess it according to the treatment patients are receiving, which could not be performed as the statistical analyses were blind to the treatment attribution.

The good psychometric properties demonstrated by the PACT-Q in this paper support its use as secondary endpoint in trials. However, one should note that key validation criteria still need to be assessed before considering its use as a primary endpoint; in particular, the assessment of the responsiveness and test-retest reliability properties of the questionnaire and the estimation of the value for the minimal important difference.

Given the design of the phase III clinical trial, test-retest reliability of the PACT-Q was not assessed. For similar reasons, it was difficult to assess the responsiveness of the questionnaire. Indeed, as the PACT-Q2 aims to assess patients' satisfaction with their treatment and treatment convenience, the largest difference that one could expect should be between patients treated with VKA and patients treated with the new anticoagulant drug. However, the scoring procedure had to be established blinded to treatment groups; comparison of patients' satisfaction between these groups could therefore not be made in this present study. Changes in satisfaction and treatment convenience, are more likely to be assessable from the treatment effect longitudinal study; these data of which will be further presented in another publication.

\section{Conclusion}

The PACT-Q is a valid and robust instrument that allows patients' expectations and satisfaction with anticoagulant treatment to be assessed. The good acceptability and psychometric properties of the questionnaire have been dem- 
onstrated with patients with various conditions including $\mathrm{DVT}, \mathrm{PE}$ and AF, suggesting the PACT-Q's suitability for thromboembolic pathologies in general. Its multi-language validated versions will facilitate and widen its use in international studies.

This instrument could be helpful for clinicians to identify patients' expectations, either positive or negative, of their anticoagulant treatment. Together with a better knowledge of how patients perceive their treatment, the questionnaire could therefore contribute to facilitate physicians' decision about the most appropriate medical care for their patients; this would probably result in a better compliance from patients, and ultimately in a better control of the disease.

\section{Competing interests}

The present work was funded by Sanofi-Aventis, Research and Development. The phase III trials were initiated by Sanofi-Aventis, Research and Development. The work on the PACT-Q was investigator-initiated. SR Kahn is supported by a Senior Clinical Investigator Award from the Fonds de la Recherche en Santé du Québec. Hélène Gilet, Isabelle Guillemin are paid consultants to Sanofi-Aventis, Research and Development. Sylvie Gabriel is an employee of Sanofi-Aventis, Research and Development. The other authors have no conflict of interest.

\section{Authors' contributions}

All authors provided intellectual contributions to this manuscript. MHP participated in the conception and design of the questionnaire, and in the data interpretation. IG participated in the data interpretation and was responsible for writing the manuscript. HG was responsible for statistical analyses and participated in the data interpretation. SG participated in the conception and the design of the questionnaire. BE provided input on the questionnaire development and data interpretation. GR contributed to the questionnaire conception and design and data interpretation. SRK provided input on the questionnaire development and data interpretation

\section{Copyrights}

PACT- $\mathrm{Q}^{\circ}$ is protected by copyright with all rights reserved to Sanofi-Aventis, France. Do not use without permission. For information on, or permission to use PACT- ${ }^{\circ}$, please contact the Mapi Research Trust, 27 rue de la Villette 69003 Lyon, France. Tel: +33 (0)4 72136575 - E-mail: trust@mapi.fr - website: http://www.mapi-trust.org.

\section{Acknowledgements}

We would like to thank Benoit Arnould (Mapi Values) for his input in the questionnaire development and data interpretation, and for reviewing the manuscript; Aude Roborel de Climens (Mapi Values) for her input in the statistical reports from which the manuscript has been written. We would also like to thank Prisca Leguet (Sanofi-Aventis) for her contribution to the development of the project. The manuscript has been reviewed for its English by Nicola Barnes, whom we would like to include in our acknowledgments.

\section{References}

I. Hirsh J, Dalen JE, Anderson DR, Poller L, Bussey H, Ansell J, Deykin D, Brandt JT: Oral anticoagulants: mechanism of action, clinical effectiveness, and optimal therapeutic range. Chest 1998, I I 4:445S-469S.

2. Ansell J, Hirsh J, Poller L, Bussey H, Jacobson A, Hylek E: The pharmacology and management of the vitamin $K$ antagonists: the seventh ACCP conference on antithrombotic and thrombolytic therapy. Chest 2004, 126:204S-233S.

3. Caprini JA, Tapson VF, Hyers TM, Waldo AL, Wittkowsky AK, Friedman R, Colgan KJ, Shillington AC: Treatment of venous thromboembolism: adherence to guidelines and impact of physician knowledge, attitudes, and beliefs. J Vasc Surg 2005, 42:726-733.

4. Ingelgard A, Hollowell J, Reddy P, Gold K, Tran K, Fitzmaurice D: What are the barriers to warfarin use in atrial fibrillation?: Development of a questionnaire. J Thromb Thrombolysis 2006, 21:257-265.

5. Dunbar-Jacob J, Erlen JA, Schlenk EA, Ryan CM, Sereika SM, Doswell WM: Adherence in chronic disease. Annu Rev Nurs Res 2000, 18:48-90.

6. Hirsh AT, Atchison JW, Berger JJ, Waxenberg LB, Lafayette-Lucey A, Bulcourf $B B$, Robinson ME: Patient satisfaction with treatment for chronic pain: predictors and relationship to compliance. Clin J Pain 2005, 21 :302-310.

7. Ware JE Jr, Davies AR: Behavioral consequences of consumer dissatisfaction with medical care. Eval Program Plann 1983, 6:291-297.

8. Waterman AD, Milligan PE, Bayer L, Banet GA, Gatchel SK, Gage BF: Effect of warfarin nonadherence on control of the International Normalized Ratio. Am J Health Syst Pharm 2004, 6I:1258-1264.

9. Woodside AG, Frey LL, Daly RT: Linking service quality, customer satisfaction, and behavioral intention. I Health Care Mark 1989, 9:5-17.

10. Oliver RL: Satisfaction: A Behavioral Perspective on the Consumer New York: McGraw-Hill; 1996.

II. Prins MH, Marrel A, Carita P, Anderson D, Bousser M-G, Crijns H, Consoli S: Multinational development of a questionnaire assessing patient satisfaction with anticoagulant treatment. Health Qual Life Outcomes 2009, 7:9. (6 Feb 2009).

12. Acquadro C, Jambon B, Ellis D, Marquis P: Language and translation issues. In Quality of Life and Pharmacoeconomics in Clinical Trials Second edition. Edited by: Spilker B. Philadelphia: Lippincott-Raven Publishers; 1996:575-585.

13. Vapnik VN: Statistical learning theory. New York: John Wiley \& Sons; 1998.

14. Fayers PM, Machin D: Factor analysis. In Quality of Life assessment in clinical trials: methods and practice Edited by: Staquet MJ, Hays RD, Fayers PM. Oxford University Press, Inc NewYork; 1998:191-223.

15. Campbell DT, Fiske DW: Convergent and discriminant validation by the multitrait-multimethod matrix. Psychol Bull 1959, 56:8I-105.

16. Hays RD, Hayashi T: Beyond internal consistency reliability: rationale and user's guide for multitrait analysis program on the microcomputer. Behav Res Methods Instrum Comput 1990, 22:167-175.

17. Cronbach LJ: Coefficient alpha and the internal structure of tests. Psychometrika 195I, 16:297-334.

18. Nunnally JC, Bernstein IH: Psychometric theory New York: McGrawHill Inc; 1994

19. Escofier B, Pagès J: Analyses factorielles simples et multiples. In Objectifs, méthodes et interprétation 3rd edition. Paris, Dunod; 1998.

20. Robert $P$, Escoufier $Y$ : A unifying tool for linear multivariate statistical methods: the RV coefficient. Applied Statistics 1976, 25:257-265.

21. Chassany O, Sagnier P, Marquis P, Fullerton S, Aaronson N: Patientreported outcomes: the example of health-related quality of life. A European guidance document for the improved integration of health-related quality of life assessment in the drug regulatory process. Drug Inf $J$ 2002, 36:209-238. 
22. Hays RD, Revicki DA, Anderson R: Psychometric considerations in evaluating health-related quality of life measures. Qual Life Res 1993, 2:441-449.

23. Scientific Advisory Committee of the Medical Outcomes Trust: Assessing health status and quality of life instruments: attributes and review criteria. Qual Life Res 2002, 2:44I-449.

24. Guyatt GH, Deyo RA, Charlson M, Levine MN, Mitchell A: Responsiveness and validity in health status measurements: a clarification. J Clin Epidemiol 1989, 42:403-408.

25. Guyatt G, Walter S, Norman G: Measuring change over time: assessing the usefulness of evaluative instruments. J Chronic Dis 1987, 40: $17 \mid-178$.

26. Hays RD, Anderson R, Revicki D: Assessing reliability and validity of measurement in clinical trials. In Quality of Life assessment in clinical trials: methods and practice Oxford University Press; 1998:169-182.

27. Kazis LE, Anderson JJ, Meenan RF: Effect sizes for interpreting changes in health status. Med Care 1989, 27:178-189.

Publish with Biomed Central and every scientist can read your work free of charge

"BioMed Central will be the most significant development for disseminating the results of biomedical research in our lifetime. "

Sir Paul Nurse, Cancer Research UK

Your research papers will be:

- available free of charge to the entire biomedical community

- peer reviewed and published immediately upon acceptance

- cited in PubMed and archived on PubMed Central

- yours - you keep the copyright

Submit your manuscript here:

http://www.biomedcentral.com/info/publishing_adv.asp
BioMedcentral 